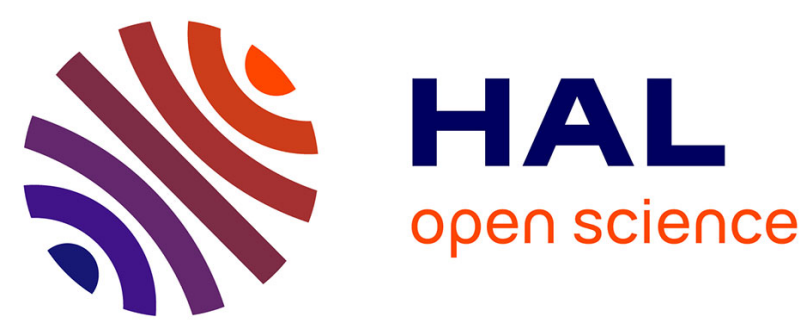

\title{
A Possible Dust Origin for an Unusual Feature in Io's Sodium Neutral Clouds
}

Cesare Grava, Timothy Cassidy, Nicholas M. Schneider, Hsiang-Wen Hsu, Jeffrey P. Morgenthaler, François Leblanc, Valeria Mangano, Kurt D. Retherford, Matthew H. Burger, Cesare Barbieri

\section{To cite this version:}

Cesare Grava, Timothy Cassidy, Nicholas M. Schneider, Hsiang-Wen Hsu, Jeffrey P. Morgenthaler, et al.. A Possible Dust Origin for an Unusual Feature in Io's Sodium Neutral Clouds. The Astronomical Journal, 2021, 162 (5), pp.190. 10.3847/1538-3881/ac1ff8 . insu-03374890

\section{HAL Id: insu-03374890 \\ https://hal-insu.archives-ouvertes.fr/insu-03374890}

Submitted on 1 Nov 2021

HAL is a multi-disciplinary open access archive for the deposit and dissemination of scientific research documents, whether they are published or not. The documents may come from teaching and research institutions in France or abroad, or from public or private research centers.
L'archive ouverte pluridisciplinaire HAL, est destinée au dépôt et à la diffusion de documents scientifiques de niveau recherche, publiés ou non, émanant des établissements d'enseignement et de recherche français ou étrangers, des laboratoires publics ou privés.

$$
\text { Copyright }
$$




\title{
A possible dust origin for an unusual feature in Io's sodium neutral clouds
}

\author{
Cesare Grava (D), 1 Timothy A. Cassidy (D), ${ }^{2}$ Nicholas M. Schneider (D), ${ }^{2}$ Hsiang-Wen Hsu (DD, ${ }^{2}$ \\ Jeffrey P. Morgenthaler (iD ${ }^{3}$ François Lebland (iD, ${ }^{4}$ Valeria Mangano (D), ${ }^{5}$ Kurt D. Retherford (D) 1,6 \\ Matthew H. Burger (iD, ${ }^{7}$ and Cesare Barbieri (iD ${ }^{8}$ \\ ${ }^{1}$ Southwest Research Institute, 6220 Culebra road, San Antonio, TX, 78238, USA \\ ${ }^{2}$ Laboratory for Atmospheric and Space Physics, University of Colorado Boulder, Boulder, CO, USA \\ ${ }^{3}$ Planetary Science Institute, Tucson, AZ, USA \\ ${ }^{4}$ LATMOS/CNRS, Sorbonne Université, UVSQ, IPSL, Paris, France \\ ${ }^{5}$ INAF/IAPS, Rome, Italy \\ ${ }^{6}$ University of Texas at San Antonio, San Antonio, TX, USA \\ ${ }^{7}$ Space Telescope Science Institute, Baltimore, MD, USA \\ ${ }^{8}$ University of Padua, Department of Physics and Astronomy, Padua, Italy
}

(Received June 1, 2019; Revised January 10, 2019; Accepted July 1, 2021)

Submitted to AJ

\begin{abstract}
We report the results of model simulations performed to explain the nature of a sodium emission feature in Io Neutral Clouds. The feature was detected via high-resolution spectroscopic observations from the 3.6-meter Italian telescope TNG. The emission feature is blueshifted compared to the main emission (the banana-shaped Neutral Cloud of Io) by a few tens of $\mathrm{km} \mathrm{s}^{-1}$, and it is most prominent when Io is a few tens of degrees before eclipse behind Jupiter's shadow. The feature's morphology changes with time, indicative of a geometrical effect. We constrained its direction, velocity, and column density with a model of sodium atom trajectories under the influence of Io's and Jupiter's gravity and solar radiation pressure. The model that best explains this emission feature has the atoms injected into the exosphere from the leading/sub-Jovian hemisphere of Io (45-68 $8^{\circ}$ West longitude), with velocities from 50 to $90 \mathrm{~km} \mathrm{~s}^{-1}$ relative to Io. These trajectories are consistent with those of negatively charged dust grains (radius $\sim 10 \mathrm{~nm}$ ) accelerated by the co-rotational electric field of Jupiter's magnetosphere. We propose that sputtering of sodium atoms from Na-bearing molecules $\left(\mathrm{NaCl}\right.$ and $\left.\mathrm{Na}_{2} \mathrm{SO}_{4}\right)$ in these nanodust grains is the process responsible for our emission feature. Both modeling and observational constraints provide an order-of-magnitude estimate of the sodium production rate of $\sim 10^{26} \mathrm{~s}^{-1}$. Our work provides another method to monitor the amount of material that Io is supplying to its Neutral Clouds and plasma torus.
\end{abstract}

Keywords: (not needed now)

\section{INTRODUCTION}

Io's Neutral Clouds are one of the brightest visible 5 manifestations of the intense volcanic and magneto${ }_{6}$ spheric activity in the Jovian system. All the material 7 that fills the Io Plasma Torus and Io's Neutral Clouds ultimately comes from the intense volcanic activity of

Corresponding author: Cesare Grava cgrava@swri.edu
39 Io. Since the discovery of sodium at Io (Brown 1974), 40 and its Neutral Cloud (Trafton et al. 1974), this mi${ }_{41}$ nor but bright species has been used to monitor the re42 sponse of Io's Neutral Cloud to the changes of its drivers ${ }_{43}$ (Io volcanic activity and Jupiter magnetosphere). The 44 resonant scattering efficiency (the so-called g-value) of 45 sodium is orders of magnitude greater than the domi46 nant species in Io's Neutral Clouds (S and $\mathrm{O}$ and their 47 molecular compounds). Moreover, the wavelength of the 48 sodium doublet (the D2 line at D1 line around $5900 \AA$ ) 49 is such that it can be easily detected from the ground. 
50 Over the past decades, observations and models have ${ }_{51}$ identified structures in the Neutral Clouds, each related 52 to a particular source process and interaction with the 53 Jovian magnetosphere. The most prominent of these 54 structures, and the first to be discovered, is the corona, 55 sometimes referred to as the "banana cloud" (the "Re56 gion B" in Brown et al. 1975), composed of slowly 57 escaping sodium atoms (a few $\mathrm{km} \mathrm{s}^{-1}$ relative to Io). 58 Its peculiar morphology, with the leading part more ex59 tended (about $60^{\circ}$ along the orbit) than the trailing part 60 (30 ${ }^{\circ}$ along the orbit), is controlled by celestial mechan${ }_{61}$ ics and by ionization from the plasma torus, which is 62 warmer outwards from Io's orbit compared to inwards 63 (Nash et al. 1986). Another feature of the sodium Neu64 tral Clouds is the "jet" (or directional feature; Goldberg 65 et al. 1984), much more variable than the banana cloud, 66 and composed of much more energetic atoms. The "jet" ${ }_{67}$ extends from Io in the anti-Jupiter direction, oriented 68 approximately perpendicularly to the local Jovian mag69 netic field (Wilson \& Schneider 1994). It is probably 70 caused by prompt pickup ion neutralization very close 71 to Io, its narrowness indicating an unperturbed Jovian magnetic field at Io (Wilson \& Schneider 1999). The 73 outer acceleration results from the corotational electric 74 field applied to positively charged ions. Finally, a more diffuse feature is the "stream" (Schneider et al. 1991) of fast moving neutral sodium atoms resulting from disso7 ciative recombination of fast molecular sodium-bearing 78 ions (most likely $\mathrm{NaCl}^{+}$) corotating with Jupiter at $\sim$ $70 \mathrm{~km} \mathrm{~s}^{-1}$. These atoms can be found all around Io's orbit. Over many orbits around Jupiter, this stream 1 creates the vast, faint neutral sodium cloud detected hundreds of Jupiter radii from Jupiter (Mendillo et al. 1990). All these features contribute to the roughly 1 ton 4 of plasma that is injected in the Io Plasma Torus every second (Schneider \& Bagenal 2007). Their morphology depends on several factors, including Io's position 7 (both orbital longitude and magnetic latitude) Jupiter's magnetic field, and Io's volcanic activity (see review by Bagenal \& Dols 2020). For example, it has long been debated whether the sodium Neutral Clouds decrease 1 in extent during eclipses behind Jupiter, due to con92 densation of Io's atmosphere and/or to a suppression of s solar photons available for photodissociation of sodium4 bearing molecular compounds. It was to test these pos5 sibilities that we performed ground-based observations 6 of Io before and after eclipses, in 2007. We discovered 9 that solar flux is an important factor in supplying the sodium atoms to the Neutral Clouds, and that suppres9 sion of photo-dissociation of sodium-bearing molecules 100 (most likely $\mathrm{NaCl}$ ) during eclipse drives a decrease in ex101 ospheric Na atoms soon after egress (Grava et al. 2014).

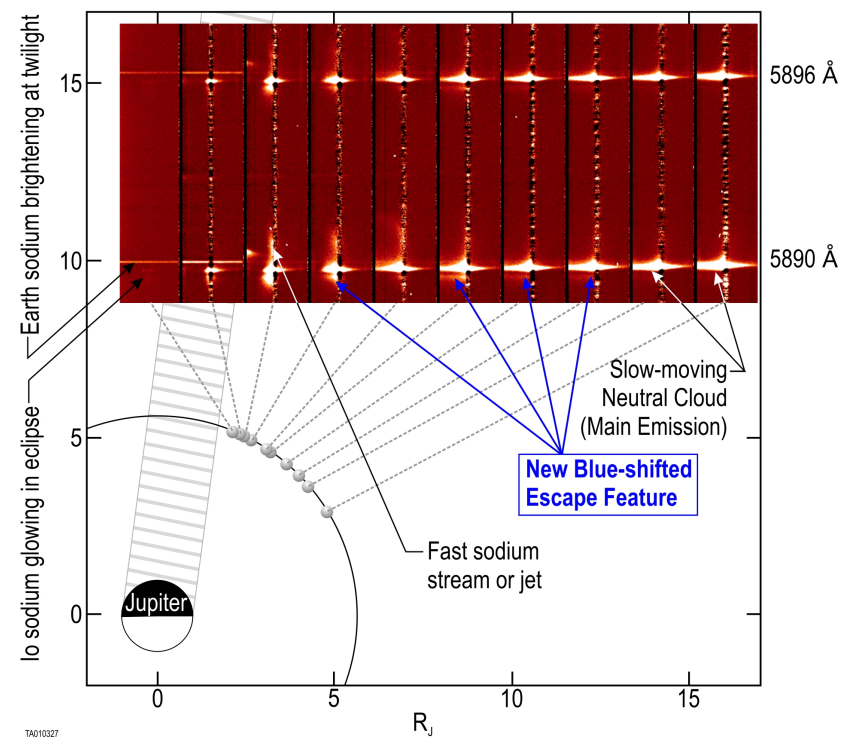

Figure 1. Two-dimensional spectra of Io with the continuum subtracted, stacked over a scheme of the observational geometry. The spectra progress in time from right to left, and wavelength run from bottom to top. The width of the spectra, corresponding to the length of the slit, is 27 arcsec. $\mathrm{R}_{\mathrm{J}}=$ Jupiter radius $\sim 70,000 \mathrm{~km}$. In the spectra, Jupiter direction is to the left of Io's subtracted continuum.

102 During those observing runs, we also discovered an 103 additional spectroscopic feature: an emission of sodium 104 (visible at both D-lines) blueshifted (i.e., directed to105 wards the observer and thus towards Jupiter) by tens 106 of $\mathrm{km} \mathrm{s}^{-1}$ relative to Io, hence moving much faster than 108 the escape speed at Io (see Figure 1). We first reported 109 this feature in Schneider et al. (2008) and performed 110 additional observations at the same telescope in 2009. 111 This paper describes the analysis of this feature using 112 simulations of sodium atoms in Io's Neutral Clouds and 113 suggests possible explanations.

\section{4 \\ 2. OBSERVATIONS AND DATA REDUCTION}

115 Spectra were collected by the now-decommissioned 116 high-resolution échelle spectrograph SARG mounted 117 at the 3.6-meter Italian telescope TNG (Telescopio ${ }_{118}$ Nazionale Galileo) during several nights spanning 2 119 years, and different geometric configurations of Io. Spec120 tra taken in 2007 were analyzed by Grava et al. (2014) 121 and included in this work for completeness, while spectra 122 taken in 2009 are analyzed here for the first time. The 123 spectrograph's slit was 26.7-arcsec long and 0.4-arcsec 124 wide, had a spectral resolution of $\lambda / \Delta \lambda \sim 115,000$, and ${ }_{125}$ was placed almost always parallel to Jupiter's rotational 126 equator and centered on Io (or on other Galilean satel127 lites, when used for calibration). The exposure time 128 was almost always 600 s. The 2007 spectra were ob- 
129 tained with an interference filter which blocked all pho130 tons but those with wavelengths close to the sodium 131 doublet. This procedure allowed to use the full extent 132 of the long slit without order overlapping. The 2009 ${ }_{133}$ spectra were obtained without such interference filter, 134 to collect light from the entire bandpass of SARG (3700$13510,000 \AA$ ) and to study therefore other species. As such, 136 only the central $\sim 50$ pixels were used, and the remain${ }_{137}$ ing $\sim 30$ pixels at the edges were excluded due to order ${ }_{138}$ contamination. The effective length of the slit in this 139 case was 17.7 arcsec.

140 Data reduction of the spectra was described in depth 141 in Grava et al. (2014) and is summarized here. Stan142 dard data reduction steps such as subtraction of bias, ${ }_{143}$ flatfielding, and wavelength calibration (using a Th-Ar 144 lamp) were performed using IRAF routines. Specific 145 data reduction steps pertinent to our dataset were per146 formed later and included: 1) removal of an interference 147 pattern caused by the sodium filter (for 2007 observa148 tions only); 2) removal of a "pedestal" (residual back149 ground); 3) removal of a "ghost" of Jupiter's spectrum 150 on top of Io spectra; 4) removal of telluric absorption, 151 performed using rapidly rotating O- and B-type stars 152 devoid of absorption lines observed at several airmasses 153 that bracketed ours; 5) subtraction of the "continuum", 154 i.e. the solar light reflected off Io's disk using spectra of 155 other Galilean satellites (Europa, Ganymede, and, more 156 rarely, Callisto) properly shifted to account for the dif157 ferent Doppler speeds; 6) conversion of sodium bright158 ness from counts $\mathrm{s}^{-1}$ to Rayleighs $\left(1 \mathrm{R}=10^{6} / 4 \pi\right.$ photons $159 \mathrm{~cm}^{-2} \mathrm{~s}^{-1} \mathrm{sr}^{-1}$; Hunten et al. (1956)) using Jupiter spec160 tra and the well-measured intrinsic brightness of Jupiter 161 at the sodium wavelength (5.5 MR $\AA^{-1}$; Brown \& Schnei162 der 1981). During most of the nights the seeing was 163 good, never exceeding 1.6 arcsec as FWHM (calculated 164 as a deconvolution of a Gaussian fit to Io's continuum 165 and the theoretical diameter of Io's disk, 1.2 arcsec). 16в Details of observations are summarized in Table 1.

\section{THE BLUESHIFTED EMISSION FEATURE}

Figure 2 shows a typical spectrum of Io. On the left 171 is the average of Io's brightness over detector columns $17231-33$, corresponding to a distance of 1.7-2.9 $\mathrm{R}_{\text {Io }}$ east${ }_{173}$ wards on the sky plane (towards Jupiter, in this case) 174 of Io's center $\left(1 \mathrm{R}_{\mathrm{Io}} \sim 1820 \mathrm{~km}\right)$ and, in dashed line, 175 the g-values. The g-value (or g-factor) is the number of 176 solar photons resonantly scattered each second by each 177 sodium atom. In optically thin exospheres, like Io's neu178 tral clouds, the brightness $I$ (in Rayleighs) is directly 179 related to the line-of-sight column density $N$ (in atoms $180 \mathrm{~cm}^{-2}$ ) by the g-value $g$ (Brown \& Yung 1976):

$$
I=g \cdot N
$$

181 The g-value (expressed in photons atoms ${ }^{-1} \mathrm{~s}^{-1}$ ) is related 182 to the solar spectrum, and depends, among other things, ${ }_{183}$ on the heliocentric radial velocity $\Delta v$ of sodium atoms, ${ }_{184}$ owing to the deep Fraunhofer absorption in the solar 185 spectrum Hunten et al. (1988). We used g-values for 186 sodium from Killen et al. (2009). $\Delta v$ is measured in ${ }_{187}$ our spectra by the Doppler shift $\Delta \lambda$ of Io relative to the ${ }_{188}$ reference wavelengths $\lambda$ of the sodium D-lines (5889.95 189 and $5895.92 \AA$ for the D2 and D1 line, respectively):

$$
\Delta v=c \cdot \frac{\Delta \lambda}{\lambda}
$$

190 where $c$ is the speed of light. The resulting $\Delta v$ is referred 191 to Io's reference frame, which is in itself moving relative 192 to the Sun. An atom at rest at Io moving away from the ${ }_{193}$ Sun (like at Western elongations) will "see" a redshifted 194 solar spectrum. Therefore, to properly convert bright195 ness into column densities it is necessary to blueshift the $196 \mathrm{~g}$-values, i.e. shifting them towards negative velocities. ${ }_{197}$ This is shown in Figure 2, panel a, which shows that 198 the "dip" in the emission line (in Rayleighs) at $-5 \mathrm{~km}$ $199 \mathrm{~s}^{-1}$ is caused by the "dip" of the Fraunhofer line of the $200 \mathrm{~g}$-value at $-2.5 \mathrm{~km} \mathrm{~s}^{-1}$. The resulting division gives the 201 column density without such "dip" (panel b), but with 202 a "bump" on the blue side of the peak (negative veloci203 ties). This is the new emission feature. It is reminiscent 204 of the "skirt" detected first by Trafton (1975) and then 205 by Cremonese et al. (1992) and interpreted by Trafton \& 206 Macy $(1977,1978)$ to be due to sodium atoms streaming 207 away from Io at moderate speeds ( $18 \mathrm{~km} \mathrm{~s}^{-1}$ at most) 208 in the leading direction. However, it is different from 209 that because the "bump" lies on the opposite side. Our ${ }_{210}$ "bump" is more prominent on the short wavelength side ${ }_{211}$ when Io is west of Jupiter. Moreover, as we shall see ${ }_{212}$ briefly, the feature changes Doppler shift through the ${ }_{213}$ night. Note that in our observations the West direction 214 corresponds to the orbital trailing side of Io. In terms 215 of plasma flow, the trailing side corresponds to the is 216 upstream side, and East is the leading (or downstream) 217 side.

${ }_{218}$ We performed the conversion from brightness to col219 umn density for all of our 2D spectra. An example of 220 the resulting $2 \mathrm{D}$ spectra, together with the geometry of 221 the observation, is shown in Figure 3 (the others can be ${ }_{223}$ found in the supplemental material). In this figure, Io 224 is about to enter Jupiter's shadow and the main emis225 sion feature (the "banana cloud"), being approximately 226 at rest relative to Io (white horizontal dashed lines), 227 dominates the spectra. The faint, straight emission line 228 traversing the whole slit length is Earth's sodium layer 229 at $90 \mathrm{~km}$ altitude resonantly scattering solar photons 230 shortly before dawn. The streak of diffuse emission very ${ }_{231}$ close to Io on the Eastern side (negative $\mathrm{R}_{\mathrm{Io}}$ ) extending 
Table 1. List of observations. $t=$ exposure time. $\mathrm{ssl}=$ subsolar longitude, counted Westward (or counterclockwise) on Io. It is also equivalent to Io's orbital longitude, with $0^{\circ}$ corresponding to superior conjunction (Io opposite to Earth behind Jupiter) and $90^{\circ}$ corresponding to Eastern elongation. $\lambda_{I I I}$ and Mlat are Io's system III longitude and magnetic latitude, respectively. p.a. = position angle, or the slit's angle relative to Jupiter's rotation axis, counted counterclockwise starting from Jupiter's north pole.

\begin{tabular}{|c|c|c|c|c|c|c|}
\hline filename & obs. mid-time (UT) & $\mathrm{t}(\mathrm{s})$ & $\operatorname{ssl}\left({ }^{\circ}\right)$ & $\lambda_{I I I}\left({ }^{\circ}\right)$ & Mlat $\left(^{\circ}\right)$ & p.a. $\left({ }^{\circ}\right)$ \\
\hline JDEI0102 & 2007-04-25T02:01:33 & 600 & 316 & 29 & -10 & 66 \\
\hline JDEI0106 & 2007-04-25Т03:07:52 & 600 & 326 & 59 & -8 & 66 \\
\hline JDEI0109 & 2007-04-25Т03:39:07 & 600 & 330 & 74 & -6 & 66 \\
\hline JDEI0112 & 2007-04-25T04:14:26 & 600 & 335 & 90 & -4 & 66 \\
\hline JDEI0114 & 2007-04-25Т04:50:26 & 600 & 340 & 107 & -1 & 66 \\
\hline JDEI0115 & 2007-04-25T05:02:42 & 600 & 342 & 112 & 0 & 66 \\
\hline JDEI0119 & 2007-04-25T05:41:37 & 600 & 347 & 130 & 3 & 90 \\
\hline JDEI0120 & 2007-04-25T05:53:17 & 600 & 349 & 136 & 4 & 90 \\
\hline JDEI0121 & 2007-04-25Т06:04:55 & 600 & 351 & 141 & 5 & 90 \\
\hline JDEI0122 & 2007-04-25Т06:12:44 & 120 & 352 & 146 & 5 & 90 \\
\hline JDWF0002 & 2007-05-11Т00:16:32 & 600 & 316 & 213 & 9 & 85 \\
\hline JDWF0004 & 2007-05-11T00:39:57 & 600 & 319 & 223 & 9 & 90 \\
\hline JDWF0008 & 2007-05-11T01:22:55 & 600 & 326 & 243 & 7 & 90 \\
\hline JDWF0010 & 2007-05-11T01:37:36 & 600 & 328 & 250 & 6 & 90 \\
\hline JDWF0015 & 2007-05-11T02:30:28 & 600 & 335 & 274 & 3 & 90 \\
\hline JDWF0017 & 2007-05-11T02:46:03 & 600 & 337 & 282 & 2 & 90 \\
\hline JDWF0022 & 2007-05-11Т03:37:23 & 600 & 345 & 305 & -2 & 90 \\
\hline JDWF0024 & 2007-05-11Т03:52:34 & 600 & 347 & 312 & -3 & 90 \\
\hline JDWF0025 & 2007-05-11T04:04:15 & 600 & 348 & 318 & -4 & 90 \\
\hline JDWF0026 & 2007-05-11T04:18:16 & 600 & 350 & 324 & -5 & 90 \\
\hline JFQI0034 & 2007-06-20T23:37:43 & 600 & 11 & 183 & 9 & 90 \\
\hline JFQI0035 & 2007-06-20T23:49:40 & 600 & 12 & 188 & 9 & 90 \\
\hline JFQI0037 & 2007-06-21T00:05:42 & 600 & 14 & 196 & 10 & 90 \\
\hline JFQI0043 & 2007-06-21T01:05:08 & 600 & 23 & 224 & 9 & 90 \\
\hline JFQI0045 & 2007-06-21T01:19:53 & 600 & 25 & 230 & 8 & 90 \\
\hline JFQI0049 & 2007-06-21T01:59:44 & 600 & 31 & 249 & 7 & 90 \\
\hline JFQI0051 & 2007-06-21T02:14:04 & 600 & 33 & 256 & 6 & 90 \\
\hline JFQI0056 & 2007-06-21T03:12:27 & 600 & 41 & 282 & 2 & 90 \\
\hline JFQI0058 & 2007-06-21T03:28:22 & 600 & 43 & 290 & 0 & 90 \\
\hline JGGV0044 & 2007-07-06T21:53:49 & 600 & 10 & 7 & -9 & 90 \\
\hline JGGV0045 & 2007-07-06T22:05:48 & 600 & 12 & 13 & -9 & 90 \\
\hline JGGV0049 & 2007-07-06T22:36:19 & 600 & 16 & 27 & -10 & 90 \\
\hline JGGV0052 & 2007-07-06T23:06:43 & 600 & 21 & 41 & -9 & 90 \\
\hline JGGV0058 & 2007-07-07T00:02:07 & 600 & 29 & 67 & -7 & 90 \\
\hline JGGV0062 & 2007-07-07T00:36:20 & 600 & 33 & 83 & -5 & 90 \\
\hline JGGV0067 & 2007-07-07T01:20:25 & 600 & 40 & 103 & -1 & 90 \\
\hline КРВB0028 & 2009-09-07T21:55:26 & 180 & 234 & 86 & -4 & -270 \\
\hline КРВB0034 & 2009-09-07T23:38:40 & 600 & 249 & 132 & 3 & -270 \\
\hline KPCB0056 & 2009-09-08T23:43:16 & 600 & 93 & 81 & -5 & -270 \\
\hline KPCB0059 & 2009-09-09Т00:24:09 & 600 & 99 & 100 & -2 & -180 \\
\hline KPFB0035 & 2009-09-09T20:22:05 & 600 & 268 & 296 & -1 & -270 \\
\hline KРFB0038 & 2009-09-09T20:59:06 & 600 & 273 & 313 & -3 & -180 \\
\hline KРFB0041 & 2009-09-09T21:33:50 & 600 & 278 & 329 & -6 & -270 \\
\hline KРFB0047 & 2009-09-09T22:27:46 & 600 & 286 & 353 & -8 & -270 \\
\hline KPIB0078 & 2009-09-12Т23:43:39 & 600 & 187 & 230 & 9 & -180 \\
\hline KPIB0081 & 2009-09-13Т00:25:12 & 600 & 193 & 249 & 7 & -270 \\
\hline KPJB0031 & 2009-09-13Т21:33:57 & 600 & 12 & 116 & 1 & -270 \\
\hline KPJB0033 & 2009-09-13Т21:51:41 & 600 & 14 & 124 & 2 & -270 \\
\hline KPJB0050 & 2009-09-14T01:30:31 & 600 & 45 & 226 & 9 & -270 \\
\hline
\end{tabular}




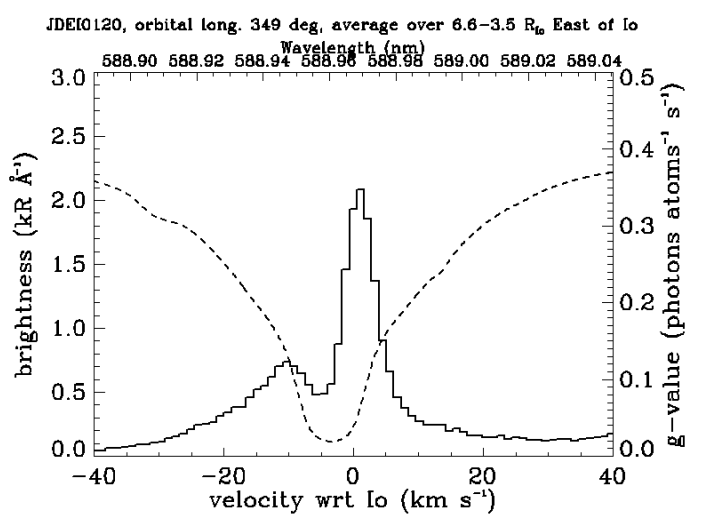

(a)

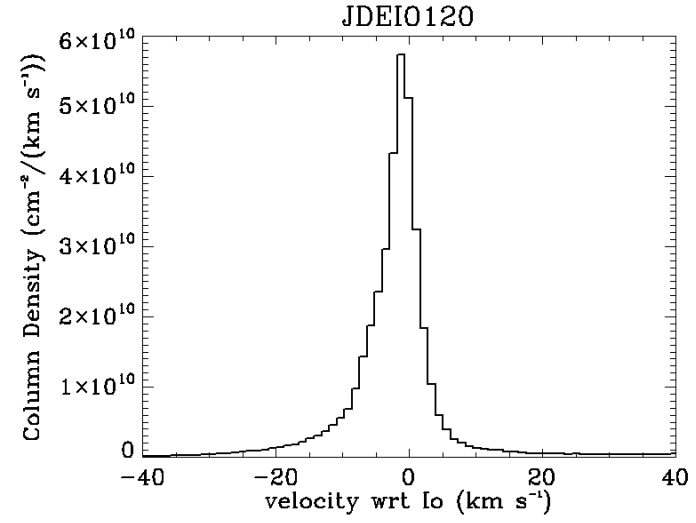

(b)

Figure 2. Left: spectrum of one Io observation (solid line) and the g-value used (dashed line) around the D2 emission line. Right: their ratio, which is the column density.

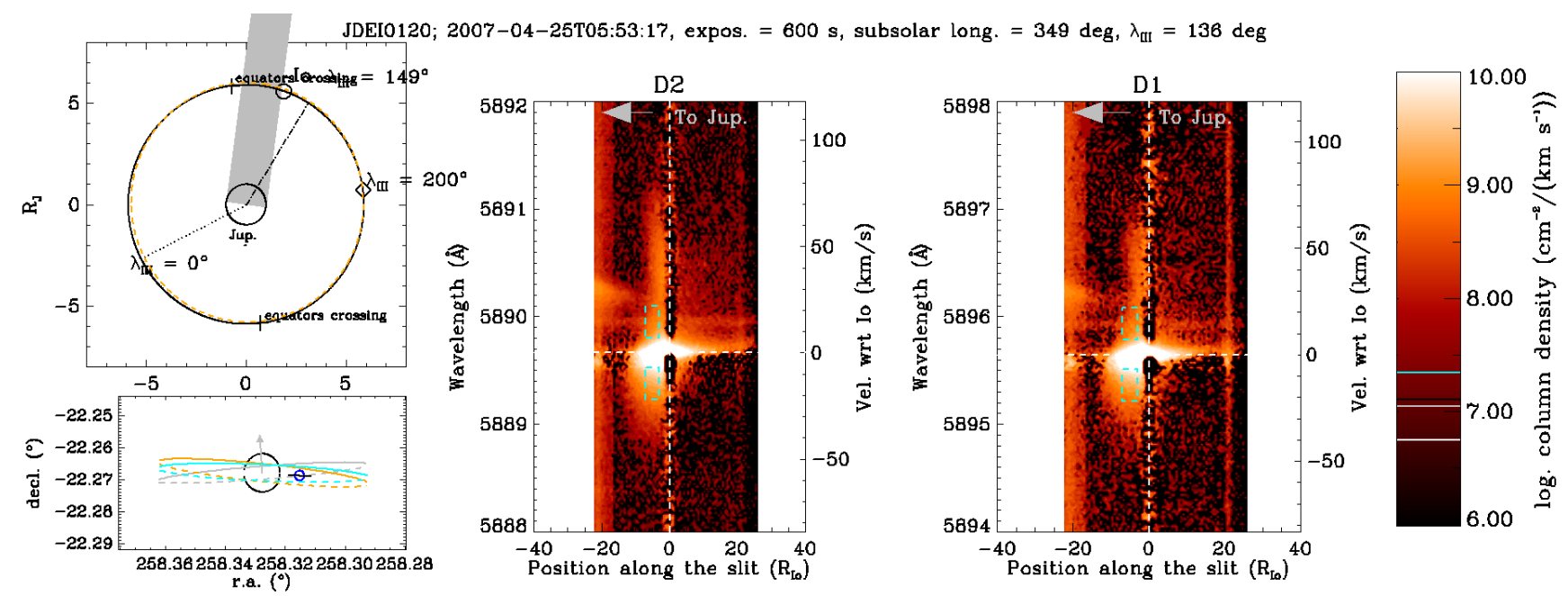

Figure 3. Composite image to put our $2 \mathrm{D}$ spectra in the observational perspective. The two right panels show our $2 \mathrm{D}$ Io spectra converted into column densities, in logarithmic color scale (D2 and D1, respectively). The region enclosed within the cyan dashed rectangles (7-22 $\mathrm{km} \mathrm{s}^{-1}$ and 3-7 Io radii) is used to compute the average source rate discussed in Section 5 and listed in Table 2. The gray arrows indicate the direction to Jupiter (towards left, in this case). The left panels show a simplified view of the geometry of the observations, from the celestial north pole (top) and from Earth (bottom), similar to Cremonese et al. (1992). Black solid circle on top (and gray on bottom) represents Io's orbit, in Jupiter's equatorial plane. Orange dashed circles on both top and bottom left panels represent Jupiter's magnetic equator at Io's distance, and include the tilt of $9.8^{\circ}$ towards System III magnetic longitude $\lambda_{I I I}=200^{\circ}$ and the offset of $0.12 \mathrm{R}_{\mathrm{J}}$ towards $\lambda_{I I I}=149^{\circ}$. Cyan circle in bottom left panel represents the centrifugal equator, where the cold plasma ions reside. The arrow in the lower left panel indicates the rotation axis of Jupiter, and is black or gray if it points towards or away from the observer, respectively. Dashed lines in lower left panel indicate portions of the circles away from the observer. The blue circle and the small black line crossing it represent Io's disk and the spectrograph's slit, respectively. All objects in the two left panels are drawn to scale, except Io, for which the size has been magnified by 10. Corresponding images for the other observations can be found in the supplemental material. 
232 to $\sim 75 \mathrm{~km} \mathrm{~s}^{-1}$ is the stream of fast sodium atoms that 233 feed the giant sodium nebula (e.g. Flynn et al. 1992). ${ }_{234}$ The feature modeled in this paper is the extended emis235 sion blueshifted by few of tens of $\mathrm{km} \mathrm{s}^{-1}$ with respect to 236 Io, meaning these sodium atoms are moving towards the 237 observer. The feature is seen here in the eastern half of 238 the slit (left, in the figure), i.e. towards Jupiter.

\section{MODELING THE EMISSION FEATURE}

240

We applied our Monte Carlo model of sodium atom 241 trajectories (Burger et al. 2014) to reproduce our obser242 vations as closely as possible, by including the location 243 and direction of ejection and the velocity distribution 244 of ejected atoms. These parameters are constrained by 245 our observations. The goal is to reproduce the emission 246 feature and its temporal variability.

${ }_{247}$ Figure 4 illustrates the change in time of the new fea248 ture's morphology at different orbital (or subsolar) lon${ }_{289}$ gitudes . This sequence shows that the blue-shifted fea251 ture, more prominent towards the end of the observing 252 run, early in the night is red-shifted by a similarly high 253 speed $\left(>10 \mathrm{~km} \mathrm{~s}^{-1}\right)$. The transition from red to blue 254 shifts suggests that $\mathrm{Na}$ is ejected in a direction that is 255 roughly perpendicular to the Io-observer line when the 256 subsolar longitude is $\sim 330^{\circ}$ (between the $4^{\text {th }}$ and the $2575^{\text {th }}$ spectrum in Figure 4). This red-to-blue shift can 258 then be explained by the changing observing geometry 259 as Io orbits Jupiter. This peculiar behavior inspired 260 our modeling approach. We first modeled the trajecto261 ries of the neutral sodium atoms (Section 4.1) and then 262 found a plausible physical mechanism that can bring 263 these sodium atoms at the location and with the speed 264 we observe them (Section 4.1).

\subsection{Neutral sodium atoms trajectories}

To simulate the observations we used the Monte Carlo 267 model that is described in Burger et al. (2014). In this 268 model, atoms are tracked under the forces of gravity and 269 solar radiation pressure. The equations of motion are 270 solved with a $5^{\text {th }}$ order, adaptive step-size Runge-Kutta 271 algorithm. Atoms are ejected at randomly selected times 272 between the start and end of the model run so that the 273 simulation contains a mixture of freshly released and 274 older atoms. Loss processes include photoionization, 275 electron-impact ionization, and charge exchange, though 276 the atoms in the simulations shown below are mostly lost 277 by leaving the small field of view. The observation ge278 ometry was recreated using the SPICE toolkit (Acton 279 1996)

We ran the model many times with different initial ${ }_{281}$ conditions in order to determine the direction and speed ${ }_{282}$ of $\mathrm{Na}$ that would reproduce the red-to-blue shift in Fig${ }_{283}$ ure 4 . Sodium atoms are ejected radially in intervals
${ }_{284} 11.25^{\circ}$ wide. The origin of the ejected atoms is labeled 285 in Io's West longitude, where $0^{\circ}$ corresponds to the sub286 Jupiter meridian and $90^{\circ}$ points along Io's orbital mo287 tion (the "leading" direction). Speeds values from 4 to ${ }_{288} 130 \mathrm{~km} \mathrm{~s}^{-1}$ were used, each with a narrow distribution 289 (each bin being $10 \mathrm{~m} \mathrm{~s}^{-1}$ wide).

290 Each of the two free parameters we used to reproduce 291 our observations affects only one aspect of the emis292 sion feature. The ejection speed distribution affects the ${ }_{293}$ Doppler shift (wavelength dimension), while the ejection 294 location affects the morphology of the emission feature 295 (spatial direction). This is illustrated in Figure 5, where 296 we show the effects of changing these parameters, com297 pared to the nominal, best model.

${ }_{298}$ We find that the best combination of parameters to re299 produce our observations is a broad speed distribution, 300 with ejection velocities relative to Io of $50-90 \mathrm{~km} \mathrm{~s}^{-1}$, 301 and a relatively narrow range of ejection location: 45$30268^{\circ}$ of Io West longitude, meaning an ejection from the 303 subjovian/leading hemisphere. Models with the nomi304 nal direction but lower speed $\left(40 \mathrm{~km} \mathrm{~s}^{-1}\right)$ or higher speed $305\left(130 \mathrm{~km} \mathrm{~s}^{-1}\right)$ fail to match the magnitude of the Doppler 306 shift, though they maintain the red-to-blue transition 307 at a subsolar point of $\sim 330^{\circ}$ (left-right panels in Fig308 ure 5). The mismatches in direction include one angular 309 bin toward the leading direction and one bin toward the 310 subjovian direction, and these both fail to reproduce the 311 red-to-blue transition and the magnitude of the Doppler 312 shift (top-down panels in Figure 5).

313 Figure 6 shows the best simulations compared to some 314 of the observations. The rest of the data-model com315 parison can be found in the movie in the Supplemental 316 Material. The nominal model shown here (in both Fig317 ure 5 and 6) includes speeds of $57 \mathrm{~km} \mathrm{~s}^{-1}$ and $90 \mathrm{~km} \mathrm{~s}^{-1}$, 318 though lower speeds could be included without signifi319 cantly changing the result. Figure 6 illustrates how our 320 model can reproduce the shift in velocity relative to Io ${ }_{321}$ (from red, or positive, to blue, or negative) within the 322 same amount of time (about 4 hours).

323 Figure 7 shows a top-down view of the nominal model 324 of sodium trajectories near the beginning and end of the 325 observing sequence. In this image, directions toward the 326 observer (Earth, $\oplus$ ) and Jupiter (4) are indicated. The 327 red-to-blue shift is seen here as a change in ejection di328 rection as seen from Earth. Also, it can be noted that 329 the ejection direction is roughly halfway, in terms of 330 degrees, between the leading direction and Jupiter di331 rection.

\section{4.2. Dust grains trajectories}

${ }_{333}$ Having found the characteristics of the sodium feature 334 (speed distribution and direction), we need to identify 

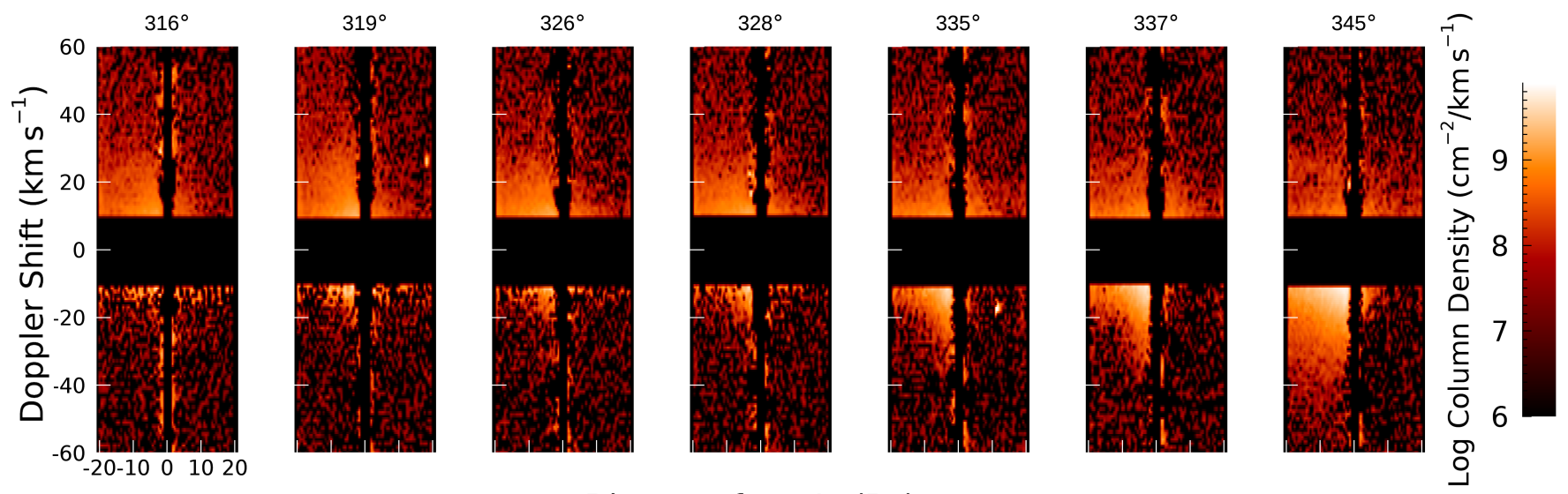

\section{Distance from lo $\left(\mathrm{R}_{10}\right)$}

Figure 4. Observations taken in May 2007, calibrated in column density per unit velocity at different orbital (or subsolar) longitudes. Doppler velocities less than $10 \mathrm{~km} \mathrm{~s}^{-1}$, which include the "banana cloud", have been blacked out to highlight the feature, which transitions from red-shifted to blue-shifted in this 4-hour time span. Io's orbital position is given as subsolar longitude and is indicated at the top of each plot. Io's continuum has been carefully removed with the procedure discussed in the text.
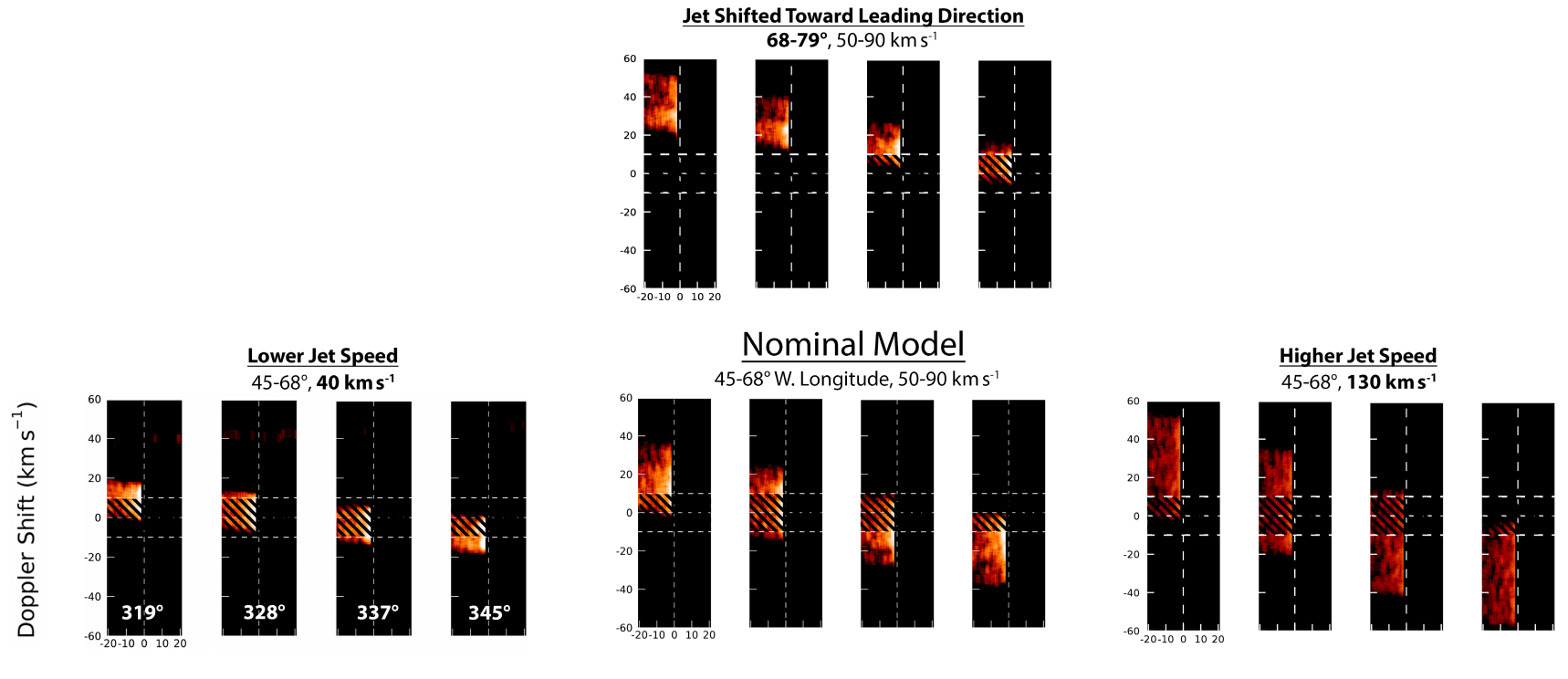

Distance from lo $\left(R_{10}\right)$

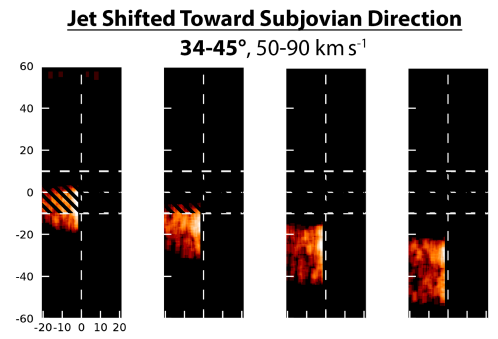

Figure 5. The simulation from the best model is shown at the center. The other four sub-panels show models with the right ejection direction but too low (left panel) or too fast (right panel) ejection speed, or models with the right ejection speed but wrong directions (top and bottom panels). Velocities within $10 \mathrm{~km} \mathrm{~s}^{-1}$, marked by horizontal dashed lines, have been masked to leave out the main emission feature. The vertical dashed line represents the region of Io's continuum. 


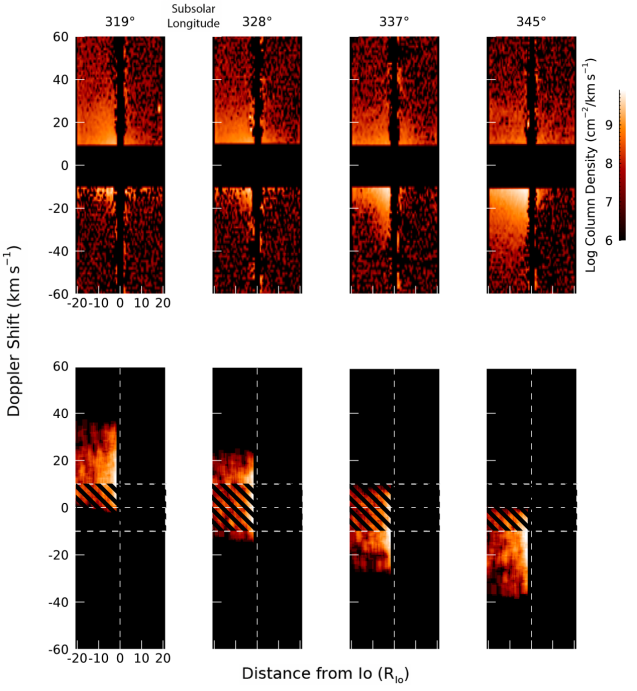

Figure 6. Comparison between four spectra in May 2007 (top panel) and their best fit model simulations (bottom panel). Note how the simulations are able to reproduce the shift in Doppler velocity, from positive relative velocities (moving away from the observer) to negative ones (moving towards the observer).
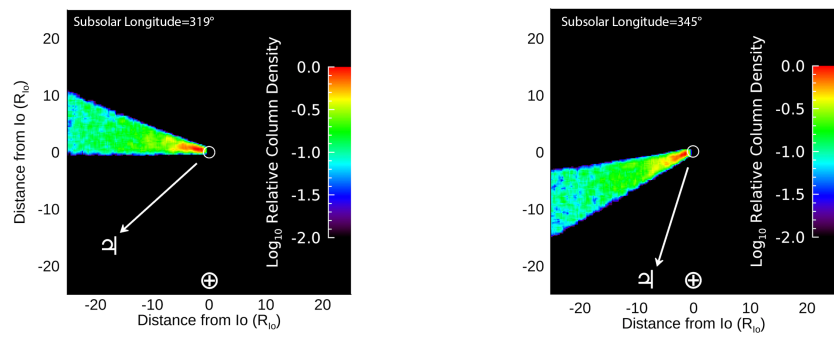

Figure 7. Top-down view of the model shown in Figure 6. The left panel shows the beginning of the observation sequence, when the $\mathrm{Na}$ is directed away from the observer to produce a red Doppler shift. The right panel shows the end of the sequence, when the $\mathrm{Na}$ is blue shifted. The white arrow points toward Jupiter, Earth is straight down.

335 a process that may bring sodium atoms at the location 336 and speed observed. The direction of the blueshifted 337 sodium feature (towards Jupiter) is consistent with neg338 atively charged particles that move following Jupiter 339 magnetosphere's co-rotational electric field (e.g. Horányi 340 et al. 1997). There are two possible candidates: sodium341 bearing negative ions that are promptly neutralized 342 and negatively charged Na-rich dust grains from which 343 sodium atoms are liberated. In this section we discuss 344 the latter, as many of the grains' properties agree with 345 our directional feature; at the end of Section 5.1 we 346 briefly discuss the negative ions hypothesis.

${ }_{347}$ Dust in the Jovian environment has been detected by 348 multiple spacecraft: Ulysses (Grün et al. 1993), Galileo
349 (Grün et al. 1996), and Cassini (Postberg et al. 2006).

350 All these observations are consistent with electrically

351 charged dust grain of radius $\sim 10 \mathrm{~nm}$ (Zook et al. 1996).

352 The periodicity of the dust impact signal in Galileo data

353 was one of the early indicators that Io, and not the gos-

354 samer rings, was the source of such dust grains (Graps

355 et al. 2000). Subsequently, the Cosmic Dust Analyser

356 (CDA) onboard Cassini was able to distinguish the com-

357 position of these grains. $\mathrm{NaCl}$ was the dominant species

358 identified by CDA, followed by other components like

$359 \mathrm{Na}_{2} \mathrm{SO}_{4}$ and $\mathrm{K}_{2} \mathrm{SO}_{4}$ (Postberg et al. 2006). This is in

360 contrast with the main composition of Io's atmosphere

361 and neutral clouds, where $\mathrm{NaCl}, \mathrm{Na}^{+}$, and $\mathrm{Cl}^{+}$are just

362 trace species $(\mathrm{NaCl}$ fraction in composition is less than

363 $1 \%$, while the $\mathrm{Na}^{+}$and $\mathrm{Cl}^{+}$concentration in the torus

364 is between 2 and 6\%; Lellouch et al. 2003; Küppers \&

365 Schneider 2000) The inverse trend in composition for

366 dust may be related to the process that ultimately ejects

367 these particles in the first place, i.e. volcanic eruptions.

368 Because of their high condensation temperature, $\mathrm{NaCl}$

369 and $\mathrm{KCl}$ are abundant condensates 20 min after out-

370 gassing from the vent, while sulphuric components, due

371 to their lower condensation temperature, are still far

372 from condensation (Zhang et al. 2004).

373 We performed simulations of the trajectory of neg-

374 atively charged nanodust grains of various sizes. We

375 assume that the dust particles $10 \mathrm{~nm}$ in radii start with

376 a circular Keplerian motion at Io's orbit and an initial

377 charge of 20 electric charge, corresponding to a surface

378 potential of $-3 \mathrm{~V}$. The charging and dynamical evolu-

379 tion of nanometer-sized grains is modeled as described

380 in Horányi et al. (1997). For individual dust grains, two

381 equations are integrated simultaneously: the equation

382 of motion and the grain charging equation. The equa-

383 tion of motion considers the gravity of Jupiter and the

${ }_{384}$ Lorentz forces acting on the grain from the co-rotating

385 magnetosphere. The grain charging equation depends

${ }_{386}$ on plasma properties at the grain's location and deter-

387 mines the grain charge-to-mass ratio, essential to cal-

388 culate the Lorentz forces. Grains can be charged neg-

389 atively or positively, depending on the plasma environ390 ment, and the charging begins almost instantaneously.

391 A top-down view of the trajectory of a negatively 392 charged dust grain of radius $10 \mathrm{~nm}$, as well as a pos393 itively charged one (same size), is shown in Figure 8. ${ }_{394}$ Figure 9 shows the direction, the relative speed and dis395 tance with respect to Io of a negatively charged dust 396 grain of radius $10 \mathrm{~nm}$. The gray circle in that figure rep397 resents the parameter space (both velocity and distance 398 to Io) consistent with our observations. The dynamical 399 evolution of charged nanoparticles largely depends on 400 the grain charge polarity: the positively charged parti- 


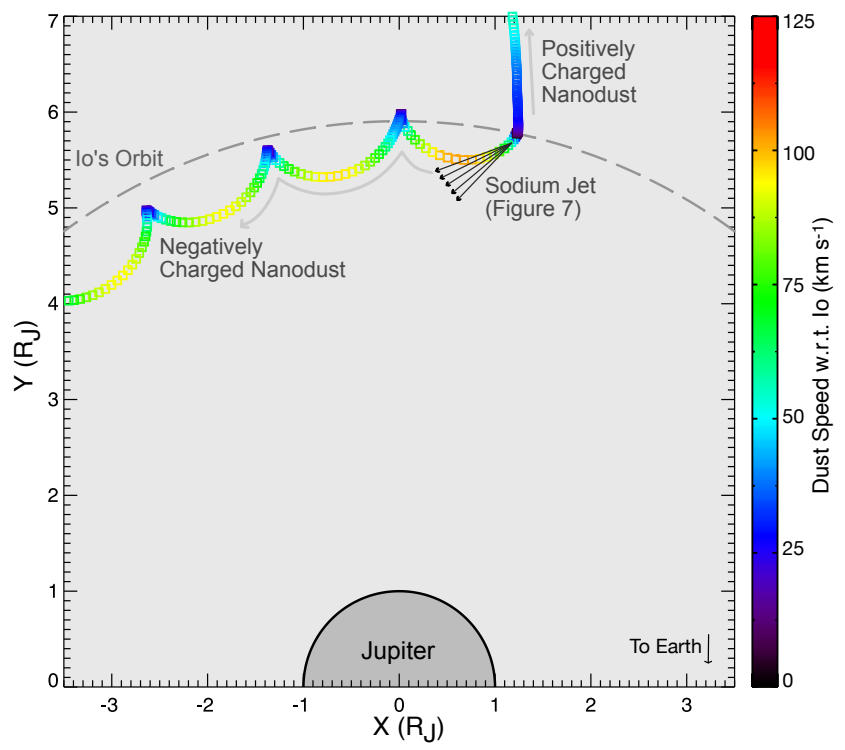

Figure 8. Top-down view of the trajectories of a negatively charged and a positively charged 10 -nm-radius dust grain. $\mathrm{R}_{\mathrm{J}}=$ Jupiter radius $\sim 70,000 \mathrm{~km}$.

401 cle is accelerated away from Jupiter in almost a straight 402 trajectory, while the negatively charged one exhibits a 403 cycloidal motion along Io's orbit similar to pick-up ions. 404 In general, negatively (positively) charged grains are ac405 celerated towards (away from) Jupiter because of the 406 outward-pointing co-rotating electric field (e.g. Horanyi 407 et al. 1993). Within the first cycloid (within the first $408 \sim 15$ minutes), when the grain is within $\sim 10$ Io radii, 409 both its direction and speed are comparable to the prop${ }_{410}$ erties of the sodium jet. Grains of larger size (e.g. 100 ${ }_{411} \mathrm{~nm}$ ), with a much smaller charge-to-mass ratio, are less ${ }_{412}$ accelerated by Lorentz forces compared to 10-nm-radius ${ }_{413}$ dust grains. Therefore, their velocity relative to Io is ${ }_{414}$ much smaller than the observed one. Similarly, much 415 smaller particles (size $<10 \mathrm{~nm}$ ) have a much smaller gy416 roradius and tend to stay much closer to Io (a couple 417 of Io radii at most) than our sodium feature. We shall ${ }_{418}$ return to this point in the next Section.

\section{DISCUSSION}

${ }_{420}$ Nanodust particles get charged at high altitude at Io ${ }_{421}$ by capture of ionospheric electrons or photoelectron pro422 duction (Krüger et al. 2003a). Only the most energetic ${ }_{423}$ plumes are expected to bring grains to high altitudes so 424 that they collect sufficient charge from ambient plasma 425 to overcome Io's gravity (Johnson et al. 1980). Flan426 des (2004) showed that particles need to reach at least ${ }_{427} \sim 400 \mathrm{~km}$ altitude to be able to escape, and the flight ${ }_{428}$ time from vent to this height is $15-20$ minutes.

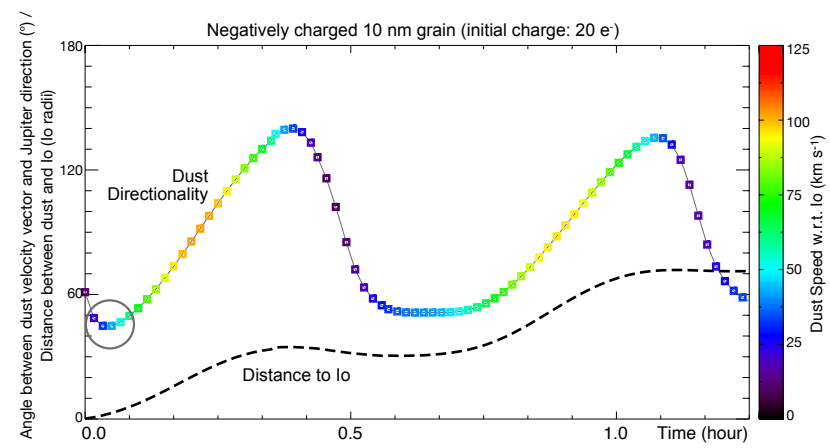

Figure 9. Simulation of trajectory of a negatively charged 10-nm-radius dust grain (of $20 \mathrm{e}^{-}$initial charge) moving under the influence the co-rotational electric field of Jupiter's magnetosphere. The line threading through the colored squares is the direction (angle), while the colored squares represent the velocity. The gray circle indicates the parameter space of the modeled neutral sodium atoms: speed range of $50-90 \mathrm{~km} \mathrm{~s}^{-1}$ (cyan to yellow colors) and direction of $\sim 45^{\circ}$. The negatively charged particles have the right velocity at the right time to match our observations. The dashed line is the distance from Io and is also referred to the y axis (in Io radii) and that is also consistent with our data (Figure 4). Tick marks on the $\mathrm{x}$ axis are 5 minutes.

${ }_{429}$ Once they get charged, these nanodust particles fol430 low trajectories that are determined by the corotational 431 electric field of Jupiter's magnetosphere, in the radial 432 direction. The dependence of dust trajectories on the 433 highly variable magnetosphere in turn implies a certain 434 variability in the expected dust flux with Jovian local 435 time even if the dust ejection rate is constant. This 436 variability, combined with the narrow field of view of ${ }_{437}$ our slit, might explain the variability seen in our dataset ${ }_{438}$ within a couple of hours (Figure 4). Most previous Io 439 nanodust detection were carried out at distances further 440 away from Io's orbit. In this respect, our observations ${ }_{441}$ probe a much closer region to Io than all previous dust 442 measurements.

${ }_{443}$ The spatial information of the observed $\mathrm{Na}$ jets also ${ }_{444}$ provides constraints on the dynamics of its possible 445 dusty source. The observed $\mathrm{Na}$ speeds correspond 446 roughly to $\sim 50 \mathrm{~km} \mathrm{~s}^{-1}$, but grains in the dust model $(10$ $447 \mathrm{~nm}$ in radius with $20 \mathrm{e}^{-}$initial charge) need to be accel${ }_{448}$ erated for some distance before reaching those speeds, ${ }_{449}$ at least $7 \mathrm{R}_{\mathrm{Io}}$ as seen from Earth.

450 A dust grain with lower initial charge gets charged 451 later, and thus farther away, and therefore its distance 452 from Io would not match our observations. Only grains ${ }_{453}$ with proper charge-to-mass ratio can be sufficiently ac454 celerated by the Lorentz forces and maintain a direction 455 comparable to the observed sodium jet. To first order, 456 regardless of the grain size, grains in the same plasma 457 environment will be charged to roughly the same elec- 
458 trostatic potential determined by the ambient plasma 459 conditions. For a given electrostatic potential, the grain 460 charge-to-mass ratio is inversely proportional to the 461 square of the grain size. Negatively charged grains much 462 larger than $10 \mathrm{~nm}$ have lower charge-to-mass ratio and 463 would not be accelerated to the observed speed in the ${ }_{464}$ vicinity of Io. Negatively charged grains much smaller 465 than $10 \mathrm{~nm}$, on the other hand, have much smaller gy466 roradius and will produce both red- and blue- shifted 467 components in the vicinity of Io, which is also not con468 sistent with the observation. The grain dynamics thus 469 suggests that the sodium jet can only be produced by 470 charged grains with radius $\sim 10 \mathrm{~nm}$. This is in good 471 agreement with previous grain size estimate of the Jo472 vian stream particles based on space dust instruments 473 on board Galileo and Cassini spacecraft (Postberg et al. 474 2006; Hsu et al. 2012).

475 It is possible to perform a rough estimate of the source 476 rate from the spectra and compare it to the source rate 477 from the neutral model. The sodium source rate $r$ is 478 the product of the peak column abundance, the vertical 479 extent $d$ of the emission, and the velocity $v_{\perp}$ perpen${ }_{480}$ dicular to the observer's line of sight (Schneider et al. 481 1991):

$$
r=d \cdot v_{\perp} \cdot \int_{v_{1}}^{v_{2}} n(v) d v
$$

${ }_{482}$ In the above formula, we used Io's diameter as verti483 cal extent $d$ of our feature; $n$ is the column density per 484 unit velocity averaged between 3.0 and $7.0 \mathrm{R}_{\text {Io }}$ and the 485 integral is performed between $v_{1}=7$ and $v_{2}=22 \mathrm{~km}$ ${ }_{486} \mathrm{~s}^{-1}$ relative to Io, where the blueshifted feature is more ${ }_{487}$ prominent. Finally, the velocity perpendicular to the ${ }_{488}$ observer's line of sight is defined as $v_{\perp}=v_{\text {los }} \cdot \tan (\theta)$, 489 where $v_{l o s}$ is the line-of-sight velocity (the one our spec490 troscopic observations are sensitive to) and $\theta$ is the angle 491 between our line of sight and the jet (we assume our di492 rectional feature, or "jet", to be the axis of the cone of ${ }_{493}$ sodium atoms - see Figure 7). This angle is necessarily 494 taken out of our modeling, since with spectroscopic ob495 servations we can only measure the radial (line of sight) 496 velocity and have no information on the true orientation 497 of the sodium feature. For each spectrum, we average ${ }_{498}$ the D2 and the D1 source rates and report these num499 bers in Table 2. The median of the resulting sodium 500 source rates is $7.0 \times 10^{25} \mathrm{Na} \mathrm{s}^{-1}$ from the data, corre501 sponding to $2.7 \mathrm{~kg} \mathrm{~s}^{-1}$. The source rate from the neutral 502 model is somehow greater $\left(2.6 \times 10^{26} \mathrm{Na} \mathrm{s}^{-1}\right.$, correspond503 ing to $10.0 \mathrm{~kg} \mathrm{~s}^{-1}$ ), but this was derived assuming a wide ${ }_{504}$ "jet" $\left(30^{\circ}\right.$ in the vertical direction). A narrower feature 505 would require proportionally less sodium.
Table 2. Sodium source rate for the emission feature discussed in this paper. The data source rate is the average of the source rates from the D2 and D1 emission lines). Model source rates for some of the April 2007 observations are not available because the emission feature was not well defined.

\begin{tabular}{lcc}
\hline \hline filename & data source rate $\left(\mathrm{s}^{-1}\right)$ & model source rate $\left(\mathrm{s}^{-1}\right)$ \\
\hline JDEI0119 & $7.0 \times 10^{25}$ & $1.6 \times 10^{26}$ \\
JDEI0120 & $1.1 \times 10^{26}$ & $2.6 \times 10^{26}$ \\
JDWF0002 & $1.4 \times 10^{25}$ & $5.8 \times 10^{25}$ \\
JDWF0004 & $2.8 \times 10^{25}$ & $9.2 \times 10^{25}$ \\
JDWF0008 & $4.6 \times 10^{25}$ & $1.4 \times 10^{26}$ \\
JDWF0010 & $7.0 \times 10^{25}$ & $2.1 \times 10^{26}$ \\
JDWF0015 & $2.3 \times 10^{26}$ & $2.9 \times 10^{26}$ \\
JDWF0017 & $1.7 \times 10^{26}$ & $2.7 \times 10^{26}$ \\
JDWF0022 & $9.8 \times 10^{25}$ & $2.8 \times 10^{26}$ \\
JDWF0024 & $7.7 \times 10^{25}$ & $3.0 \times 10^{26}$ \\
JDWF0025 & $6.9 \times 10^{25}$ & $2.2 \times 10^{26}$ \\
\hline
\end{tabular}

${ }_{507}$ For the dust hypothesis to work, we need a mechanism 508 that releases sodium atoms from these nanodust grains. 509 Possible processes include photon-stimulated desorption 510 (PSD), electron-stimulated desorption (ESD), or ion 511 sputtering. The first two have too low a source rate. ${ }_{512}$ From experiments of PSD of $\mathrm{Na}$ from surfaces that sim513 ulate the lunar silicates (Yakshinskiy \& Madey 1999), 514 scaling the solar flux to Jupiter's distance, and taking 515 into account our derived distribution of dust grains, the 516 source rate from PSD is $10^{9} \mathrm{~s}^{-1}$. The same authors also 517 showed that electron-stimulated desorption has an only 518 slightly higher cross section compared to PSD. The other 519 mechanism, ion sputtering, gives higher source rates, al520 though there are several uncertainties that affect the 521 calculation of the rate. First, an absolute value for 522 sputtering yield of sodium atoms has only been mea${ }_{523}$ sured on $\mathrm{Na}_{2} \mathrm{~S}$ : Chrisey et al. (1988) report a sputtering ${ }_{524}$ yield of 0.1 and $0.2 \mathrm{Na}_{2} \mathrm{~S}$ ion ${ }^{-1}$ from $\mathrm{O}^{+}$and $\mathrm{S}^{+}$ions, 525 respectively. The molecules of interest in our case are 526 sodium chloride $(\mathrm{NaCl})$ and sodium sulfate $\left(\mathrm{Na}_{2} \mathrm{SO}_{4}\right)$, 527 given that these were the compositions of the Na-bearing 528 dust grains detected by Cassini CDA (Postberg et al. 529 2006). Sodium sputtering yield for $\mathrm{NaCl}$ has been esti530 mated by Johnson (2000) to be $\sim 1.0 \mathrm{Na}^{-1}{ }^{-1}$, assum531 ing that $\mathrm{NaCl}$ has the same atomic ejection efficiency ${ }_{532}$ as $\mathrm{Na}_{2} \mathrm{~S}$. Sodium sputtering yield for $\mathrm{Na}_{2} \mathrm{SO}_{4}$ has only 533 been reported as a relative value: it is 100-1000 times 534 greater than the sputtering yield for $\mathrm{NaS}$ (Wiens et al. 535 1997). Assuming the absolute sputtering yield of NaS 536 to be the same as that of $\mathrm{Na}_{2} \mathrm{~S}$, we can estimate the ${ }_{537} \mathrm{Na}$ sputtering yield from $\mathrm{Na}_{2} \mathrm{SO}_{4}$ to be 100-1000 times 538 greater than that of $\mathrm{NaCl}$, or $100-1000 \mathrm{Na}$ ion $^{-1}$. The 
539 flux $\Phi$ of precipitating ions responsible for the sputtering 540 (mostly $\mathrm{S}^{+}$and $\mathrm{O}^{+}$) can be calculated by multiplying 541 the velocity of the ions (co-rotation, or $57 \mathrm{~km} \mathrm{~s}^{-1}$ ) by 542 the density of these heavy ions close to Io, or about 1500 $543 \mathrm{~cm}^{-3}$ (Dougherty et al. 2017). We obtain $\Phi=8.6 \times 10^{9}$ $544 \mathrm{~cm}^{-2} \mathrm{~s}^{-1}$.

${ }_{545}$ The second uncertainty is the dust available for sput546 tering. Over the course of the 7-year mission and from 547 a distance between 13 and $400 \mathrm{R}_{\mathrm{J}}$ from Io, the Galileo ${ }_{548}$ Dust Detector System (DDS) measured dust production 549 rates at Io between $10^{-3}$ and $10^{2} \mathrm{~kg} \mathrm{~s}^{-1}$ (with an average 550 value between 0.1 and $1.0 \mathrm{~kg} \mathrm{~s}^{-1}$ ), possibly correlated 551 to volcanic eruptions at Io (Figure 2 of Krüger et al. 552 2003b).

${ }_{553}$ With these numbers, we can estimate the number of ${ }_{554} \mathrm{Na}$ atoms sputtered by nanodust grains and compare it 555 to our observed sodium production rate. We start by 556 assuming a dust production rate of $1 \mathrm{~kg} \mathrm{~s}^{-1}$, the up557 per value of the average range reported by Krüger et al. 558 (2003b). Assuming a spherical shape and a density of $5591.5 \mathrm{~g} \mathrm{~cm}^{-3}$ (Krüger et al. 2003b), this corresponds to $5601.6 \times 10^{20}$ grains $\mathrm{s}^{-1}$ of radius $10 \mathrm{~nm}$. We now multiply 561 this number by the time it takes for the dust grains to 562 reach the point where we do see sodium emission, or 0.05 ${ }_{563}$ hours (from Figure 9), to get $N_{\text {grains }}=2.9 \times 10^{22}$. The 564 total area available for sputtering will be $N_{\text {grains }} \cdot A \cdot c$, 565 where $A$ is the area of a $10-$ nm-radius spherical nanodust 566 grain, and $c$ the concentration of either $\mathrm{NaCl}(90 \%)$ 567 or $\mathrm{Na}_{2} \mathrm{SO}_{4}(10 \%)$, from Cassini CDA measurements ${ }_{568}$ (Postberg et al. 2006). These areas are $3.2 \times 10^{11} \mathrm{~cm}^{-2}$ 569 and $3.6 \times 10^{10} \mathrm{~cm}^{-2}$ for $\mathrm{NaCl}$ and $\mathrm{Na}_{2} \mathrm{SO}_{4}$, respectively. 570 Multiplying them by the corresponding sputtering yields ${ }_{571} Y$ and the heavy ion flux, we get the sodium production 572 rate for sputtering $s$ :

$$
s=N_{\text {grains }} \cdot A \cdot c \cdot \Phi \cdot Y
$$

${ }_{573}$ We obtain $2.8 \times 10^{21} \mathrm{Na} \mathrm{s}^{-1}$ from NaCl , and $3.1 \times 10^{22}$ ${ }_{574} \mathrm{Na} \mathrm{s}^{-1}$ from $\mathrm{Na}_{2} \mathrm{SO}_{4}$ from $1 \mathrm{~kg} \mathrm{~s}^{-1}$ of dust material.

575 These numbers are between 3 and 4 orders of mag576 nitude lower than our inferred rate of $7.0 \times 10^{25} \mathrm{~s}^{-1}$ 577 (from the observations). They can be reconciled with 578 our values if we assume some combination of the high579 est estimate for dust production rate from Galileo (100 $580 \mathrm{~kg} \mathrm{~s}^{-1}$ ), the highest estimate for the sputtering yield of ${ }_{581} \mathrm{Na}$ from $\mathrm{Na}_{2} \mathrm{SO}_{4}\left(1000 \mathrm{Na}\right.$ ion $\left.{ }^{-1}\right)$, and a surface area 582 for porous particles which is $\sim 10$ times higher than the 583 spherical area (this is the case for micron-sized lunar 584 regolith particles; Taylor \& Meek 2004). Additionally, 585 the dust production rates from Galileo should be con${ }_{586}$ sidered a lower limit, as dust is likely to undergo other ${ }_{587}$ destruction processes (not included in the calculations ${ }_{588}$ of Krüger et al. 2003b) between Io and the distance at
${ }_{589}$ which grains were detected by Galileo (between 13 and ${ }_{590} 400 \mathrm{R}_{\mathrm{J}}$ from Io). This is particularly true if some ejected 591 dust has a more volatile composition than $\mathrm{NaCl}$. Finally, 592 it is not clear how the ion sputtering experiments, per593 formed on flat surfaces, would change if the target is 594 composed of nm-sized porous surfaces. To further in595 vestigate the discrepancy, a detailed calculation of the 596 release of $\mathrm{Na}$ from dust particles and full treatment of 597 dust destruction in Jupiter's highly complicated plasma 598 environment would be required, and these are outside 599 the scope of this paper.

600 We note that the new sodium feature was most promi601 nent when Io was within $5^{\circ}$ of the magnetic equator (Ta602 ble 1). The bulk of the Io plasma torus is confined in ${ }_{603}$ the centrifugal equator, which is very close to the mag604 netic one. The plasma environment of that region (high 605 plasma electron density and low electron temperature; 606 Meyer-Vernet et al. 1995) is consistent with the negative 607 grain charge polarity inferred from the trajectory simu608 lation. In addition, the sputtering erosion responsible to 609 release sodium atoms from dust surfaces is also higher at 610 low magnetic latitudes because of the higher ion density. ${ }_{611}$ Our results thus indicate an indirect pathway to deliver 612 sodium atoms, and likely other species, from Io to the ${ }_{613}$ Neutral Clouds (and ultimately to the magnetosphere) 614 by sputtering from dust surfaces.

${ }_{615}$ We conclude this section by evaluating and discarding ${ }_{616}$ another possible candidate, mentioned in Section 4.2, 617 that is a population of negative ions formed inside Io's ${ }_{618}$ plumes. In this case, the most likely process to create ${ }_{619}$ these ions is three-body electron attachment. The most 620 likely species to undergo this process is $\mathrm{NaSO}_{4}$, a radical ${ }_{621}$ species with a substantial electron affinity and the high622 est rate coefficient among several Na-bearing species. ${ }_{623}$ The negative ions would then release the neutral sodium 624 atoms we observe following electron detachment. The 625 concentration of $\mathrm{NaSO}_{4}$ inside Io's plumes is unknown, ${ }_{626}$ but to match our observed source rate per unit volume, 627 a density similar to that of potassium would be required, ${ }_{628}$ or 9 orders of magnitude greater than the the expected 629 concentration of $\mathrm{Na}_{2} \mathrm{SO}_{4}$ inside Io's plumes (Moses et al. 630 2002). For this reason, we favor the hypothesis of ion ${ }_{631}$ sputtering from negatively charged dust nanograins as ${ }_{632}$ the most plausible source of our new sodium emission 633 feature.

\section{4}

635 We have performed Monte Carlo simulation of sodium 636 atoms under the influence of Io's gravity and solar ra637 diation pressure to explain an unusual Jupiter-oriented 638 feature (Schneider et al. 2008) we detected in our high639 resolution spectra of Io's sodium Neutral Clouds from 
640 the TNG telescope. This feature is directed towards ${ }_{641}$ Jupiter and is rapidly variable in time. The best model 642 is the one that has the sodium atoms ejected in the 643 leading-sub/Jovian hemisphere of Io (45-68 West lon644 gitude) with a broad velocity distribution $(50-90 \mathrm{~km}$ $\left.{ }_{645} \mathrm{~s}^{-1}\right)$. We propose that the mechanism most likely re646 sponsible for creating sodium atoms with that orienta${ }_{647}$ tion and that speed is sputtering of $\mathrm{Na}$ from $\mathrm{Na}$-bearing 648 molecules $\left(\mathrm{NaCl}\right.$ or $\mathrm{Na}_{2} \mathrm{SO}_{4}$, both detected near Jupiter ${ }_{649}$ by Cassini CDA) attached to negatively charged dust 650 grains (10 $\mathrm{nm}$ in radius) that move under the influence 651 of the co-rotational electric field of Jupiter's magneto652 sphere. This is consistent with modeling of trajectories 653 of negatively charged nanodust grains, which present the 654 right velocity and orientation consistent with our ob655 servations and our sodium model. The median sodium 656 source rate inferred from our observations is $7.0 \times 10^{25}$ ${ }_{657} \mathrm{Na} \mathrm{s}^{-1}$, to be compared with the theoretical estimate 658 from ion sputtering of $\mathrm{Na}$ from $\mathrm{NaCl}$ or $\mathrm{Na}_{2} \mathrm{SO}_{4}$ be659 tween $5.5 \times 10^{21} \mathrm{Na} \mathrm{s}^{-1}$ and $6.2 \times 10^{26} \mathrm{Na} \mathrm{s}^{-1}$, depending ${ }_{660}$ on the choice of several uncertain parameters, such as ${ }_{661}$ the sputtering yield, the dust production rate at Io, and ${ }_{662}$ surface area available to sputtering. We point out the ${ }_{663}$ need for detailed calculations of the release of $\mathrm{Na}$ from ${ }_{664}$ dust particles and full treatment of dust destruction in ${ }_{665}$ Jupiter's highly complicated plasma environment.

${ }_{666}$ Our results uncover a new mechanism by which Io's 667 sodium Neutral Clouds are replenished, and highlight ${ }_{668}$ the need for future observing campaigns to better con669 strain the escape rate of sodium atoms produced at Io 670 by this mechanism, before the arrival of Europa Clip671 per spacecraft, whose dust counter will be able to study 672 the dust population in the Jupiter environment at much 673 closer range than any other spacecraft so far.

674

675 676 677 678 679 680 681 682 683 684 685 686 687 688 689 690 for observations in 2007 can be requested to the corre691 sponding author.

\section{REFERENCES}

692 Acton, C. H. 1996, Planet. Space Sci., 44, 65,

693 doi: 10.1016/0032-0633(95)00107-7

694 Bagenal, F., \& Dols, V. 2020, Journal of Geophysical

695 Research (Space Physics), 125, e27485,

696 doi: 10.1029/2019JA027485

697 Brown, R. A. 1974, in IAU Symposium, Vol. 65,

700 Brown, R. A., Goody, R. M., Murcray, F. J., \& Chaffee,

701 F. H., J. 1975, ApJL, 200, L49, doi: 10.1086/181894

702 Brown, R. A., \& Schneider, N. M. 1981, Icarus, 48, 519,

703 doi: 10.1016/0019-1035(81)90061-0

704 Brown, R. A., \& Yung, Y. L. 1976, in Jupiter, 1102-1145

705 Burger, M. H., Killen, R. M., McClintock, W. E., et al.

706 2014, Icarus, 238, 51, doi: 10.1016/j.icarus.2014.04.049

707 Chrisey, D. B., Johnson, R. E., Boring, J. W., \& Phipps,

708 J. A. 1988, Icarus, 75, 233,

709 doi: 10.1016/0019-1035(88)90003-6

710 Cremonese, G., Thomas, N., Barbieri, C., \& Pernechele, C.

$711 \quad 1992$, A\&A, 256, 286
712 Dougherty, L. P., Bodisch, K. M., \& Bagenal, F. 2017,

713 Journal of Geophysical Research (Space Physics), 122,

714 8257, doi: 10.1002/2017JA024053

715 Flandes, A. 2004, Geophys. Res. Lett., 31, L16802,

716 doi: $10.1029 / 2004$ GL020046

717 Flynn, B., Mendillo, M., \& Baumgardner, J. 1992, Icarus,

718 99, 115, doi: 10.1016/0019-1035(92)90176-8

719 Goldberg, B. A., Garneau, G. W., \& Lavoie, S. K. 1984,

720 Science, 226, 512, doi: 10.1126/science.226.4674.512

721 Graps, A. L., Grün, E., Svedhem, H., et al. 2000, Nature,

722 405, 48, doi: 10.1038/35011008

${ }_{723}$ Grava, C., Schneider, N. M., Leblanc, F., et al. 2014,

724 Journal of Geophysical Research (Planets), 119, 404,

725 doi: 10.1002/2013JE004504

726 Grün, E., Zook, H. A., Baguhl, M., et al. 1993, Nature, 362,

727 428, doi: 10.1038/362428a0

728 Grün, E., Baguhl, M., Hamilton, D. P., et al. 1996, Nature,

729 381, 395, doi: 10.1038/381395a0

730 Horányi, M., Grün, E., \& Heck, A. 1997,

731 Geophys. Res. Lett., 24, 2175, doi: 10.1029/97GL01539 
732 Horanyi, M., Morfill, G., \& Grun, E. 1993, Nature, 363,

733 144, doi: 10.1038/363144a0

734 Hsu, H.-W., Krüger, H., \& Postberg, F. 2012, in Nanodust

735 in the Solar System: Discoveries and Interpretations

736 (Springer), 77-117

737 Hunten, D. M., Morgan, T. H., \& Shemansky, D. E. 1988,

738 The Mercury atmosphere., ed. F. Vilas, C. R. Chapman,

739 \& M. S. Matthews, 562-612

740 Hunten, D. M., Roach, F. E., \& Chamberlain, J. W. 1956,

741 Journal of Atmospheric and Terrestrial Physics, 8, 345,

742 doi: 10.1016/0021-9169(56)90111-8

743 Johnson, R. E. 2000, Icarus, 143, 429,

744 doi: $10.1006 /$ icar.1999.6327

745 Johnson, T. V., Morfill, G., \& Grun, E. 1980,

746 Geophys. Res. Lett., 7, 305,

747 doi: 10.1029/GL007i005p00305

748 Killen, R., Shemansky, D., \& Mouawad, N. 2009, ApJS,

749 181, 351, doi: 10.1088/0067-0049/181/2/351

750 Krüger, H., Horányi, M., \& Grün, E. 2003a,

751 Geophys. Res. Lett., 30, 1058,

752 doi: 10.1029/2002GL015920

753 Krüger, H., Geissler, P., Horányi, M., et al. 2003b,

754 Geophys. Res. Lett., 30, 2101,

755 doi: 10.1029/2003GL017827

756 Küppers, M., \& Schneider, N. M. 2000,

757 Geophys. Res. Lett., 27, 513, doi: 10.1029/1999GL010718

758 Lellouch, E., Paubert, G., Moses, J. I., Schneider, N. M., \&

759 Strobel, D. F. 2003, Nature, 421, 45,

760 doi: 10.1038/nature01292

761 Mendillo, M., Baumgardner, J., Flynn, B., \& Hughes, W. J.

762 1990, Nature, 348, 312, doi: 10.1038/348312a0

763 Meyer-Vernet, N., Moncuquet, M., \& Hoang, S. 1995,

764 Icarus, 116, 202, doi: 10.1006/icar.1995.1121

765 Moses, J. I., Zolotov, M. Y., \& Fegley, B. 2002, Icarus, 156,

766 107, doi: 10.1006/icar.2001.6759

767 Nash, D. B., Yoder, C. F., Carr, M. H., Gradie, J., \&

768 Hunten, D. M. 1986, Io, ed. J. A. Burns \& M. S.

769 Matthews, 629-688
770 Postberg, F., Kempf, S., Srama, R., et al. 2006, Icarus, 183, $771 \quad$ 122, doi: 10.1016/j.icarus.2006.02.001

772 Schneider, N. M., \& Bagenal, F. 2007, Io's neutral clouds, 773 plasma torus, and magnetospheric interaction, ed.

774 R. M. C. Lopes \& J. R. Spencer, 265,

775 doi: 10.1007/978-3-540-48841-5_11

776 Schneider, N. M., Grava, C., \& Barbieri, C. 2008, in

777 AAS/Division for Planetary Sciences Meeting Abstracts

778 \#40, AAS/Division for Planetary Sciences Meeting

779 Abstracts, 59.09

780 Schneider, N. M., Trauger, J. T., Wilson, J. K., et al. 1991,

781 Science, 253, 1394, doi: 10.1126/science.253.5026.1394

782 Taylor, L. A., \& Meek, T. T. 2004, in International Lunar

783 Conference 2003, ed. S. M. Durst, C. T. Bohannan, C. G.

784 Thomason, M. R. Cerney, \& L. Yuen, Vol. 108, 109

785 Trafton, L. 1975, ApJL, 202, L107, doi: 10.1086/181991

786 Trafton, L., \& Macy, W., J. 1977, ApJ, 215, 971,

787 doi: $10.1086 / 155433$

788 Trafton, L., \& Macy, W. 1978, Icarus, 33, 322,

789 doi: 10.1016/0019-1035(78)90152-5

790 Trafton, L., Parkinson, T., \& Macy, W., J. 1974, ApJL,

791 190, L85, doi: 10.1086/181512

792 Wiens, R. C., Burnett, D. S., Calaway, W. F., et al. 1997,

${ }_{793}$ Icarus, 128, 386, doi: 10.1006/icar.1997.5758

794 Wilson, J. K., \& Schneider, N. M. 1994, Icarus, 111, 31,

795 doi: 10.1006/icar.1994.1131

796 —. 1999, J. Geophys. Res., 104, 16567,

797 doi: 10.1029/1999JE900017

798 Yakshinskiy, B. V., \& Madey, T. E. 1999, Nature, 400, 642,

799 doi: $10.1038 / 23204$

800 Zhang, J., Goldstein, D. B., Varghese, P. L., et al. 2004,

801 Icarus, 172, 479, doi: 10.1016/j.icarus.2004.06.016

802 Zook, H. A., Grun, E., Baguhl, M., et al. 1996, Science,

803274,1501 , doi: 10.1126/science.274.5292.1501 